\title{
Existence and uniqueness of solutions to complex-valued nonlinear impulsive differential systems
}

Tao Fang ${ }^{1,2}$ and Jitao Sun ${ }^{1 *}$

"Correspondence: sunjt@sh163.net 'Department of Mathematics, Tongji University, Shanghai, 200092, China

Full list of author information is available at the end of the article

\begin{abstract}
Since the quantum system, a classical example of complex-valued system, is one of the foci of ongoing research, in this paper, the issue of existence and uniqueness of solutions to nonlinear impulsive differential systems defined in complex fields, to be brief, complex-valued nonlinear impulsive differential systems, is addressed. The existence and uniqueness conditions of solutions of such systems are established by fixed point theory.

MSC: 34A37; 34A12; 34A34

Keywords: nonlinear system; impulsive system; existence and uniqueness; solution; complex
\end{abstract}

\section{Introduction}

Impulsive differential equations have become more important in recent years in some mathematical models of real processes and phenomena studied in physics, chemical technology, population dynamics, biotechnology and economics. Nowadays, there has been increasing interest in the analysis and synthesis of impulsive systems, or impulsive control systems, due to their theoretical and practical significance, for example [1-7] and the references therein.

As the fundamental issues of modern impulse theory, the existence and uniqueness of solutions to impulsive differential systems have been studied extensively in recent years, especially in the area of impulsive differential equations with fixed moments, see the monographs of Lakshmikantham et al. [7], Samoilenko and Perestyuk [8], the literature [9, $10]$ and references therein. However, the common setting adopted in the above-mentioned works is always in real number fields. In fact, equations of many classical systems such as Schrödinger equation [11], Ginzburg-Landau equation [12], Riccati equation [13] and OrrSommerfeld equation [14] are considered in the complex number fields. But, there have been few reports about the analysis and synthesis of complex dynamical systems, for example, [15-22] and references therein. More complex than the real system, the study on complex dynamical systems has many potential applications in science and engineering. For example, recently, research on the control theory of quantum systems has attracted considerable attention [17-19]. Quantum systems are a class of complex dynamical systems which take values in a Banach space in a complex field. Another example of complex dynamical systems is complex-valued neural networks. Complex-valued neural networks

(c) 2012 Fang and Sun; licensee Springer. This is an Open Access article distributed under the terms of the Creative Commons Attribution License (http://creativecommons.org/licenses/by/2.0), which permits unrestricted use, distribution, and reproduction in any medium, provided the original work is properly cited. 
have been found highly useful in extending the scope of applications in optoelectronics, filtering, imaging, speech synthesis, computer vision, remote sensing, quantum devices, spatio-temporal analysis of physiological neural devices and systems, and artificial neural information processing [20, 21].

Although the controllability and observability of complex-valued differential systems have been discussed in the papers [15] and [16], to the best of our knowledge, there has been no result so far about the existence and uniqueness of solutions to complex-valued nonlinear impulsive differential systems. The issue of the existence and uniqueness of solutions to complex-valued differential system, which is the premise and foundation when discussing this system, is one of the most fundamental ones. So it is very important and necessary to study the existence and uniqueness of solutions to complex-valued nonlinear impulsive differential systems. Due to these reasons, in this paper, we consider the fundamental concepts of solutions of the complex-valued nonlinear impulsive differential systems by an algebraic approach. The main difficulty is to investigate the conditions for existence and uniqueness of solution of complex-valued nonlinear impulsive differential systems in the context of complex fields. Explicit characterization for existence and uniqueness of this kind of system is presented by Banach's fixed point theorem. This problem is meaningful and challenging.

The paper is organized as follows. In Section 2, the complex-valued nonlinear impulsive differential systems to be dealt with are formulated and several results about the space of the complex-valued continuous functions are presented. The conditions for existence and uniqueness of solution of complex-valued nonlinear impulsive differential systems are also established in Section 2. An example that illustrates the main points of the paper is presented in Section 3. Finally, some conclusions are drawn in Section 4.

\section{Existence and uniqueness results}

In this section, we will give the existence and uniqueness of solutions to complex-valued nonlinear impulsive differential systems. First, we introduce notations, definitions, and preliminary facts which are used throughout this paper.

By $C(J, \mathbb{R})$ we denote the Banach space of all continuous functions from interval $J$ into $\mathbb{R}$ with the norm

$$
\|x(t)\|=\sup \{|x(t)|: t \in J\} .
$$

By $C\left(J, \mathbb{R}^{n}\right)$ we denote the Banach space of all continuous functions from interval $J$ into $\mathbb{R}^{n}$ with the norm

$$
\|x(t)\|_{J R}=\left\{\sum_{i=1}^{n}\left\|x_{i}(t)\right\|^{2}\right\}^{1 / 2},
$$

where $x_{i}(t)$ is the $i$ th component of $x(t)$. By $C\left(J, \mathbb{C}^{n}\right)$ we denote the space of all complex functions defined by

$$
C\left(J, \mathbb{C}^{n}\right)=\left\{x(t) \mid x_{k}(t)=\operatorname{Re}\left(x_{k}(t)\right)+i \operatorname{Im}\left(x_{k}(t)\right): \operatorname{Re}\left(x_{k}(t)\right), \operatorname{Im}\left(x_{k}(t)\right) \in C(J, \mathbb{R})\right\},
$$

where $k=1,2, \ldots, n, i=\sqrt{-1}, x_{k}(t)$ denotes the $k$ th component of $x(t), \operatorname{Re}\left(x_{k}(t)\right)$ and $\operatorname{Im}\left(x_{k}(t)\right)$ are the real part and imaginary part of $x_{k}(t)$, respectively. 
By $P C\left(J, \mathbb{C}^{n}\right)$ we denote the space of all complex piecewise functions defined by

$$
\begin{aligned}
P C\left(J, \mathbb{C}^{n}\right)= & \left\{x(t) \mid x(t)=\operatorname{Re}(x(t))+i \operatorname{Im}(x(t)): \operatorname{Re}\left(x\left(t_{k}^{-}\right)\right), \operatorname{Re}\left(x\left(t_{k}^{+}\right)\right),\right. \\
& \operatorname{Im}\left(x\left(t_{k}^{-}\right)\right) \text {and } \operatorname{Im}\left(x\left(t_{k}^{+}\right)\right) \text {exist with } \operatorname{Re}\left(x\left(t_{k}^{-}\right)\right)=\operatorname{Re}\left(x\left(t_{k}\right)\right), \\
& \left.\operatorname{Im}\left(x\left(t_{k}^{-}\right)\right)=\operatorname{Im}\left(x\left(t_{k}\right)\right), \text { and } \operatorname{Re}\left(x_{k}(t)\right), \operatorname{Im}\left(x_{k}(t)\right) \in C\left(J_{k}, \mathbb{R}^{n}\right)\right\},
\end{aligned}
$$

where $k=0,1,2, \ldots, m, t \in J=\left[t_{0}, T\right], t_{0}<t_{1}<t_{2}<\cdots<t_{m}<T$, and $J_{0}=\left[t_{0}, t_{1}\right], J_{l}=\left(t_{l}, t_{l+1}\right]$, $l=1,2, \ldots, m-1, J_{m}=\left(t_{m}, T\right]$.

We are now able to define a complex-valued nonlinear impulsive differential system on the interval $J=\left[t_{0}, T\right]$,

$$
\begin{cases}\dot{z}(t)=f(t, z(t)), & t \in J, t \neq t_{k}, \\ z\left(t_{k}^{+}\right)-z\left(t_{k}\right)=I_{k}\left(z\left(t_{k}^{-}\right)\right), & t=t_{k}, \\ z\left(t_{0}^{+}\right)=z_{0}, & \end{cases}
$$

where $t_{0}<t_{1}<t_{2}<\cdots<t_{m}<T, z(t) \in P C\left(J, \mathbb{C}^{n}\right), f: J \times \Omega \mapsto \mathbb{C}^{n}$ is a given function, $\Omega \subset \mathbb{C}^{n}$ is a closed set. $I_{k} \in C\left(\mathbb{C}^{n}, \mathbb{C}^{n}\right), k=1,2, \ldots, m$.

The solution of the complex-valued nonlinear impulsive differential system can be defined as follows.

Definition 2.1 A function $z(t) \in P C\left(\left[t_{0}, t_{0}+T\right], \mathbb{C}^{n}\right)$ is said to be a solution of $(1)$, if $z(t)$ satisfies

$$
\begin{cases}\dot{z}(t)=f(t, z(t)), & t \in\left[t_{0}, t_{0}+T\right], t \neq t_{k}, \\ z\left(t_{k}^{+}\right)-z\left(t_{k}\right)=I_{k}\left(z\left(t_{k}^{-}\right)\right), & t=t_{k}, \\ z\left(t_{0}^{+}\right)=z_{0} . & \end{cases}
$$

In the sequel we shall need the properties of a complex-valued function space, which we prove for reader's convenience.

Lemma 2.2 $C\left(J, \mathbb{C}^{n}\right)$ is a Banach space in the field $\mathbb{R}$ with the norm

$$
\|x(t)\|_{J C}=\left(\|\operatorname{Re}(x(t))\|_{J R}^{2}+\|\operatorname{Im}(x(t))\|_{J R}^{2}\right)^{1 / 2} .
$$

Proof Let $x(t)$ and $y(t)$ be arbitrary two functions of $C\left(J, \mathbb{C}^{n}\right), \alpha \in \mathbb{R}$.

Step $1\|\cdot\|_{J C}$ is a norm of $C\left(J, \mathbb{C}^{n}\right)$.

(i) $\|x(t)\|_{J C} \geq 0$, and $\|x(t)\|_{J C}=0 \Longleftrightarrow\|\operatorname{Re}(x(t))\|_{J R}=\|\operatorname{Im}(x(t))\|_{J R}=0 \Longleftrightarrow \operatorname{Re}(x(t))=$ $\operatorname{Im}(x(t))=0 \Longleftrightarrow x(t)=0$.

(ii) $\|\alpha x(t)\|_{J C}=\left(\|\operatorname{Re}(\alpha x(t))\|_{J R}^{2}+\|\operatorname{Im}(\alpha x(t))\|_{J R}^{2}\right)^{1 / 2}=$ $\left(\|\alpha \operatorname{Re}(x(t))\|_{J R}^{2}+\|\alpha \operatorname{Im}(x(t))\|_{J R}^{2}\right)^{1 / 2}=|\alpha|\|x(t)\|_{J C}$.

(iii)

$$
\begin{aligned}
\|x(t)+y(t)\|_{J C} & =\left(\|\operatorname{Re}(x(t)+y(t))\|_{J R}^{2}+\|\operatorname{Im}(x(t)+y(t))\|_{J R}^{2}\right)^{1 / 2} \\
& =\left(\|\operatorname{Re}(x(t))+\operatorname{Re}(y(t))\|_{J R}^{2}+\|\operatorname{Im}(x(t))+\operatorname{Im}(y(t))\|_{J R}^{2}\right)^{1 / 2}
\end{aligned}
$$




$$
\begin{aligned}
\leq & {\left[\left(\|\operatorname{Re}(x(t))\|_{J R}+\|\operatorname{Re}(y(t))\|_{J R}\right)^{2}\right.} \\
& \left.+\left(\|\operatorname{Im}(x(t))\|_{J R}+\|\operatorname{Im}(y(t))\|_{J R}\right)^{2}\right]^{1 / 2} \\
\leq & \left(\|\operatorname{Re}(x(t))\|_{J R}^{2}+\|\operatorname{Im}(x(t))\|_{J R}^{2}\right)^{1 / 2} \\
& +\left(\|\operatorname{Re}(y(t))\|_{J R}^{2}+\|\operatorname{Im}(y(t))\|_{J R}^{2}\right)^{1 / 2} \\
= & \|x(t)\|_{J C}+\|y(t)\|_{J C^{\circ}} .
\end{aligned}
$$

So $\|\cdot\|_{J C}$ is a norm of $C\left(J, \mathbb{C}^{n}\right)$.

Step $2\left(C\left(J, \mathbb{C}^{n}\right),\|\cdot\|_{J C}\right)$ is a complete space.

Let $\left\{x^{(s)}\right\}$ be an arbitrary Cauchy series, then for arbitrary $\varepsilon>0$, exists $N>0$, when $s, l>N$,

$$
\left\|x^{(s)}-x^{(l)}\right\|_{J C}<\varepsilon
$$

namely

$$
\left(\left\|\operatorname{Re}\left(x^{(s)}\right)-\operatorname{Re}\left(x^{(l)}\right)\right\|_{J R}^{2}+\left\|\operatorname{Im}\left(x^{(s)}\right)-\operatorname{Im}\left(x^{(l)}\right)\right\|_{J R}^{2}\right)^{1 / 2}<\varepsilon .
$$

So

$$
\left\|\operatorname{Re}\left(x^{(s)}\right)-\operatorname{Re}\left(x^{(l)}\right)\right\|_{J R}^{2}<\varepsilon, \quad\left\|\operatorname{Im}\left(x^{(s)}\right)-\operatorname{Im}\left(x^{(l)}\right)\right\|_{J R}^{2}<\varepsilon,
$$

the inequality (3) shows that $\operatorname{Re}\left(\left\{x^{(s)}\right\}\right)$ and $\operatorname{Im}\left(\left\{x^{(s)}\right\}\right)$ are the Cauchy series of $C\left(J, \mathbb{R}^{n}\right)$, since $C\left(J, \mathbb{R}^{n}\right)$ is a Banach space, $\operatorname{Re}\left(\left\{x^{(s)}\right\}\right)$ and $\operatorname{Im}\left(\left\{x^{(s)}\right\}\right)$ must be convergent. Hence $\left\{x^{(s)}\right\}$ is convergent. The proof is completed.

Lemma 2.3 $P C\left(J, \mathbb{C}^{n}\right)$ is a Banach space in the field $\mathbb{R}$ with the norm

$$
\|x(t)\|_{P C}=\max \left\{\|x(t)\|_{J_{k} C}, k=0,1, \ldots, m\right\},
$$

where $\|x(t)\|_{J_{k} C}$ is the norm of $x(t)$ which is restricted in $J_{k}$.

Proof Let $x(t)$ and $y(t)$ be arbitrary two functions of $P C\left(J, \mathbb{C}^{n}\right), \alpha \in \mathbb{R}$.

Step $1\|\cdot\|_{P C}$ is a norm of $P C\left(J, \mathbb{C}^{n}\right)$.

(i) $\|x(t)\|_{P C} \geq 0$, and $\|x(t)\|_{P C}=0 \Longleftrightarrow\|x(t)\|_{J_{k} C}=0, k=0,1, \ldots, m \Longleftrightarrow x(t)=0$.

(ii) $\|\alpha x(t)\|_{P C}=\max \left\{\|\alpha x(t)\|_{J_{k} C}\right\}=\max \left\{|\alpha|\|x(t)\|_{J_{k} C}\right\}=|\alpha| \max \left\{\|x(t)\|_{J_{k} C}\right\}=$ $|\alpha|\|x(t)\|_{P C}$.

(iii)

$$
\begin{aligned}
\|x(t)+y(t)\|_{P C} & =\max \left\{\|x(t)+y(t)\|_{J_{k} C}\right\} \leq \max \left\{\|x(t)\|_{J_{k} C}+\|y(t)\|_{J_{k} C}\right\} \\
& \leq \max \left\{\|x(t)\|_{J_{k} C}\right\}+\max \left\{\|y(t)\|_{J_{k} C}\right\} \\
& =\|x(t)\|_{P C}+\|y(t)\|_{P C} .
\end{aligned}
$$

So $\|\cdot\|_{P C}$ is a norm of $P C\left(J, \mathbb{C}^{n}\right)$.

Step $2\left(P C\left(J, \mathbb{C}^{n}\right),\|\cdot\|_{P C}\right)$ is a complete space. 
Let $\left\{x^{(s)}\right\}$ be an arbitrary Cauchy series in $P C\left(J, \mathbb{C}^{n}\right)$, then for arbitrary $\varepsilon>0$, exists $N>0$, when $s, l>N$,

$$
\left\|x^{(s)}-x^{(l)}\right\|_{P C}<\varepsilon
$$

namely

$$
\max \left\{\left\|x^{(s)}-x^{(l)}\right\|_{J_{k} C}\right\}<\varepsilon .
$$

So, for every $k$, we have

$$
\left\{\left\|x^{(s)}-x^{(l)}\right\|_{J_{k} C}\right\}<\varepsilon,
$$

the inequality (4) shows that the restriction functions of $x^{(s)}$ in $J_{k}$ are the Cauchy series of $C\left(J, \mathbb{C}^{n}\right)$. By Lemma 2.2, $C\left(J, \mathbb{C}^{n}\right)$ is a Banach space, the restriction functions of $x^{(s)}$ in $J_{k}$ must be convergent. Hence $\left\{x^{(s)}\right\}$ is convergent. The proof is completed.

Theorem 2.4 Let $f: J \times \Omega \rightarrow \mathbb{C}^{n}$ be a continuous function. Then $z(t)$ is the unique solution of the initial value problem (1) if and only if $z(t)$ is a solution of impulsive integral equation

$$
z(t)=z_{0}+\int_{t_{0}}^{t} f(s, z(s)) d s+\sum_{t_{0}<t_{k}<t} I_{k}\left(z\left(t_{k}^{-}\right)\right) .
$$

Proof Let $z(t)$ be a possible solution of the complex impulsive system (1), then $z(t)$ is a solution to

$$
\dot{z}(t)=f(t, z(t)),
$$

for $t \in J:=\left[t_{0}, T\right], t \neq t_{k}$. Assume that $t_{k}<t<t_{k+1}, k=1,2, \ldots, m$. The integration of above equality yields

$$
\begin{gathered}
z\left(t_{1}^{-}\right)-z\left(t_{0}^{+}\right)=\int_{t_{0}}^{t_{1}} f(s, z(s)) d s, \\
z\left(t_{2}^{-}\right)-z\left(t_{1}^{+}\right)=\int_{t_{1}}^{t_{2}} f(s, z(s)) d s, \\
z\left(t_{3}^{-}\right)-z\left(t_{2}^{+}\right)=\int_{t_{2}}^{t_{3}} f(s, z(s)) d s, \\
\vdots \\
z(t)-z\left(t_{k}^{+}\right)=\int_{t_{k}}^{t} f(s, z(s)) d s .
\end{gathered}
$$

Adding these together, we get

$$
\begin{aligned}
z(t)= & z_{0}+\int_{t_{0}}^{t} f(s, z(s)) d s+\left[\left(z\left(t_{1}^{+}\right)-z\left(t_{1}^{-}\right)\right)\right. \\
& \left.+\left(z\left(t_{2}^{+}\right)-z\left(t_{2}^{-}\right)\right)+\cdots+\left(z\left(t_{k}^{+}\right)-z\left(t_{k}^{-}\right)\right)\right]
\end{aligned}
$$




$$
\begin{aligned}
& =z_{0}+\int_{t_{0}}^{t} f(s, z(s)) d s+\sum_{i=1}^{k} I_{i}\left(z\left(t_{i}^{-}\right)\right) \\
& =z_{0}+\int_{t_{0}}^{t} f(s, z(s)) d s+\sum_{t_{0}<t_{k}<t} I_{k}\left(z\left(t_{k}^{-}\right)\right) .
\end{aligned}
$$

The above equality shows that $z(t)$ is a solution to (5).

Assume that $z(t)$ satisfies the integration equation (5), obviously

$$
\dot{z}(t)=f(t, z(t)),
$$

for $t \in J:=\left[t_{0}, T\right], t \neq t_{k}$.

When $t=t_{2}$, by (5)

$$
\begin{aligned}
z\left(t_{2}\right) & =z_{0}+\int_{t_{0}}^{t_{2}} f(s, z(s)) d s+\sum_{t_{0}<t_{k}<t_{2}} I_{k}\left(z\left(t_{k}^{-}\right)\right) \\
& =z_{0}+\int_{t_{0}}^{t_{1}} f(s, z(s)) d s+\int_{t_{1}}^{t_{2}} f(s, z(s)) d s+I_{1}\left(z\left(t_{1}^{-}\right)\right) \\
& =z_{0}+z\left(t_{1}^{-}\right)-z_{0}+z\left(t_{2}^{-}\right)-z\left(t_{1}^{+}\right)+I_{1}\left(z\left(t_{1}^{-}\right)\right) .
\end{aligned}
$$

Thus $z\left(t_{1}^{+}\right)-z\left(t_{1}^{-}\right)=I_{1}\left(z\left(t_{1}^{-}\right)\right)$. By the same way, we can easily prove that $z\left(t_{k}^{+}\right)-z\left(t_{k}\right)=$ $I_{k}\left(z\left(t_{k}^{-}\right)\right), k=1,2, \ldots, m$. The proof is completed.

We are now in a position to state and prove the existence and uniqueness result of the complex impulsive system (1).

Theorem 2.5 Assume that the following hypotheses hold:

(i) There exists $m(t) \in L^{1}\left(\left[t_{0}, T\right], R^{+}\right)$such that

$$
\left|f\left(t, z_{1}\right)-f\left(t, z_{2}\right)\right| \leq m(t)\left|z_{1}-z_{2}\right|
$$

for all $t \in\left[t_{0}, t_{0}+T\right], z_{1}, z_{2} \in \Omega \subset \mathbb{C}^{n}$.

(ii) There exist constants $c_{k} \geq 0, k=1,2, \ldots, m$, such that

$$
\left|I_{k}\left(z_{1}\right)-I_{k}\left(z_{2}\right)\right| \leq c_{k}\left|z_{1}-z_{2}\right|
$$

for each $z_{1}, z_{2} \in \Omega \subset \mathbb{C}^{n}$, and $\sum_{k=1}^{m} c_{k}<1$,

where $|\cdot|$ is the Euclid norm of $\mathbb{C}^{n}$. Then the complex impulsive system (1) has a unique solution in $\left[t_{0}, t_{0}+T\right]$.

Proof Transform the problem (1) into a fixed point problem. Consider the map $H$ : $P C\left(J, \mathbb{C}^{n}\right) \rightarrow P C\left(J, \mathbb{C}^{n}\right)$ defined by

$$
H(z(t))=z\left(t_{0}^{+}\right)+\int_{t_{0}}^{t} f(s, z(s)) d s+\sum_{t_{0}<t_{k}<t} I_{k}\left(z\left(t_{k}^{-}\right)\right) .
$$


We shall show that $H$ is a contraction. For arbitrary $z_{1}(t), z_{2}(t) \in P C\left(J, \mathbb{C}^{n}\right)$, then we have for each $t \in J$

$$
\begin{aligned}
& \left|H\left(z_{1}(t)\right)-H\left(z_{2}(t)\right)\right| \\
& \quad \leq \int_{t_{0}}^{t}\left|f\left(s, z_{1}(s)\right)-f\left(s, z_{2}(s)\right)\right| d s+\sum_{t_{0}<t_{k}<t}\left|I_{k}\left(z_{1}\left(t_{k}\right)\right)-I_{k}\left(z_{2}\left(t_{k}\right)\right)\right| \\
& \quad \leq \int_{t_{0}}^{t} m(s)\left|z_{1}(s)-z_{2}(s)\right| d s+\sum_{t_{0}<t_{k}<t} c_{k}\left|z_{1}\left(t_{k}\right)-z_{2}\left(t_{k}\right)\right| \\
& \quad \leq \int_{t_{0}}^{t} m(s) e^{\tau M(s)} e^{-\tau M(s)}\left|z_{1}(s)-z_{2}(s)\right| d s+\sum_{t_{0}<t_{k}<t} c_{k} e^{\tau M(t)} e^{-\tau M(t)}\left|z_{1}\left(t_{k}\right)-z_{2}\left(t_{k}\right)\right| \\
& \quad \leq \int_{t_{0}}^{t} m(s) e^{\tau M(s)}\left\|z_{1}(s)-z_{2}(s)\right\|_{B P C} d s+\sum_{t_{0}<t_{k}<t} c_{k} e^{\tau M(t)}\left\|z_{1}(t)-z_{2}(t)\right\|_{B P C} \\
& \quad \leq \int_{t_{0}}^{t} \frac{1}{\tau}\left(e^{\tau M(s)}\right)^{\prime}\left\|z_{1}(s)-z_{2}(s)\right\|_{B P C} d s+\sum_{t_{0}<t_{k}<t} c_{k} e^{\tau M(t)}\left\|z_{1}(t)-z_{2}(t)\right\|_{B P C} \\
& \quad \leq\left(\frac{1}{\tau} e^{\tau M(t)}+\sum_{t_{0}<t_{k}<t} c_{k} e^{\tau M(t)}\right)\left\|z_{1}(t)-z_{2}(t)\right\|_{B P C}
\end{aligned}
$$

Thus

$$
\left\|H\left(z_{1}(t)\right)-H\left(z_{2}(t)\right)\right\|_{P C} \leq\left(\frac{1}{\tau} e^{\tau M(t)}+\sum_{t_{0}<t_{k}<t} c_{k} e^{\tau M(t)}\right)\left\|z_{1}(t)-z_{2}(t)\right\|_{B P C}
$$

where $M(t)=\int_{t_{0}}^{t} m(s) d s$ and $\tau$ is sufficiently large and $\|\cdot\|_{B P C}$ is the norm on $P C\left(J, \mathbb{C}^{n}\right)$ defined by

$$
\|z(t)\|_{B P C}=\left\|e^{-\tau M(t)} z(t)\right\|_{P C} .
$$

Therefore,

$$
\left\|H\left(z_{1}(t)\right)-H\left(z_{2}(t)\right)\right\|_{B P C} \leq\left(\frac{1}{\tau}+\sum_{t_{0}<t_{k}<t} c_{k}\right)\left\|z_{1}(t)-z_{2}(t)\right\|_{B P C} .
$$

We can always choose $\tau$, such that $1 / \tau+\sum_{t_{0}<t_{k}<t} c_{k}<1$, so $H$ is a contraction. By the Lemma 2.3, Theorem 2.4 and the Banach contraction principle, $H$ has a unique fixed point which is a solution to (1). The proof is completed.

\section{Example}

Example 3.1 Let $W=\{z|| z \mid \leq \rho\}, \rho>0, J_{1}=[0, \pi / 2], J_{2}=(\pi / 2, \pi], J=J_{1} \cup J_{2}$, the existence and uniqueness of complex-valued nonlinear impulsive system

$$
\begin{cases}\dot{z}(t)=z^{2} \cos t, & t \in J, t \neq \pi / 2, \\ z\left(\pi / 2^{+}\right)-z(\pi / 2)=1, & t=\pi / 2, \\ z\left(0^{+}\right)=i, & \end{cases}
$$

are considered on $J \times W$. 
Obviously the condition (ii) of Theorem 2.4 is satisfied. Moreover, for arbitrary $z, w \in W$

$$
|f(t, z)-f(t, w)|=|\cos t|\left|z^{2}-w^{2}\right| \leq 2 \rho|z-w|
$$

so the hypothesis of the Theorem 2.5 holds. By Theorem 2.5, the complex-valued impulsive system (6) has a unique solution. In fact, the solution of the system (6) is

$$
z(t)= \begin{cases}\frac{i-\sin t}{1+\sin ^{2} t}, & t \in J_{1}, \\ \frac{2-\sin t+i}{1+(\sin t-2)^{2}}, & t \in J_{2} .\end{cases}
$$

\section{Conclusion}

In this paper, the issue on the existence and uniqueness of the complex-valued nonlinear impulsive system has been addressed for the first time. Taking advantage of the differential equation theory in complex fields, the existence and uniqueness conditions for such systems have been established without imposing extra conditions.

\section{Competing interests}

The authors declare that they have no competing interests.

\section{Authors' contributions}

TF completed the proof and wrote the initial draft. JS provided the problem and gave some suggestions on the amendment. TF then finalized the manuscript. Correspondence was mainly done by JS. Both authors read and approved the final manuscript.

\section{Author details}

${ }^{1}$ Department of Mathematics, Tongji University, Shanghai, 200092, China. ${ }^{2}$ School of Fundamental Studies, Shanghai University of Engineering Science, Shanghai, 201620, China.

\section{Acknowledgements}

The authors would like to thank the editor and the reviewers for their constructive comments and suggestions which improved the quality of the paper. This work is supported by the NNSF of China under Grant 61174039, and by the Fundamental Research Funds for the Central Universities of China.

Received: 18 May 2012 Accepted: 4 July 2012 Published: 20 July 2012

\section{References}

1. Lisena, B: Dynamical behavior of impulsive and periodic Cohen Grossberg neural networks. Nonlinear Anal. 74 4511-4519 (2011)

2. Chen, WH, Zheng, WX: Input-to-state stability for networked control systems via an improved impulsive system approach. Automatica 47(4), 789-796 (2011)

3. Li, DS, Long, SJ: Attracting and quasi-invariant sets for a class of impulsive stochastic difference equations. Adv. Differ. Equ. 2011, 3 (2011)

4. Li, CX, Sun, JT, Sun, RY: Stability analysis of a class of stochastic differential delay equations with nonlinear impulsive effects. J. Franklin Inst. 347(7), 1186-1198 (2010)

5. Wang, JR, Xiang, X, Wei, W: Bounded and periodic solutions of semilinear impulsive periodic system on Banach spaces. Fixed Point Theory Appl. 2008, 401947 (2008)

6. Zhang, Y, Sun, JT: Stability of impulsive delay differential equations with impulses at variable times. Dyn. Syst. 20, 323-331 (2005)

7. Lakshmikantham, V, Bainov, DD, Simeonov, PS: Theory of Impulsive Differential Equations. World Scientific, Singapore (1989)

8. Samoilenko, AM, Perestyuk, NA: Impulsive Differential Equations. World Scientific, Singapore (1995)

9. Ouahab, A: Local and global existence and uniqueness results for impulsive functional differential equations with multiple delay. J. Math. Anal. Appl. 323, 456-472 (2006)

10. Ahmad, B, Alsaedi, A: Existence and uniqueness of solutions for coupled systems of higher-order nonlinear fractional differential equations. Fixed Point Theory Appl. 2010, 364560 (2010)

11. Kato, T: On nonlinear Schrödinger equations. Ann. Inst. Henri Poincaré, a Phys. Théor. 46(1), 113-129 (1987)

12. Doering, C, Gibbon, J, Holm, D, Nicolaenko, B: Low-dimensional behaviour in the complex Ginzburg-Landau equation. Nonlinearity 1(2), 279-309 (1988)

13. Ráb, M: Geometrical approach to the study of the Riccati differential equation with complex-valued coefficients. J. Differ. Equ. 25(1), 108-114 (1977)

14. Orszag, SA: Accurate solution of the Orr-Sommerfeld stability equation. J. Fluid Mech. 50(4), 659-703 (1971) 
15. Zhao, SW, Sun, JT: Controllability and observability for impulsive systems in complex fields. Nonlinear Anal. 11, 1513-1521 (2010)

16. Fu, XY: Null controllability for the parabolic equation with a complex principal part. J. Funct. Anal. 257, 1333-1354 (2009)

17. Barreiro, JT, Müller, M, Schindler, P, Nigg, D, Monz, T, Chwalla, M, Hennrich, M, Roos, CF, Zoller, P, Blatt, R: An open-system quantum simulator with trapped ions. Nature 470, 486-491 (2011)

18. Schoelkopf, RJ, Girvin, SM: Wiring up quantum systems. Nature 451, 664-669 (2008)

19. Nielsen, MA, Chuang, L: Quantum Computation and Quantum Information. Cambridge University Press, Cambridge (2000)

20. Rahime, C, Murat, C, Yuksel, O: Fuzzy clustering complex-valued neural network to diagnose cirrhosis disease. Expert Syst. Appl. 38(8), 9744-9751 (2011)

21. Hirose, A: Complex-Valued Neural Networks: Theories and Applications. World Scientific, Singapore (2003)

22. Everitt, WN, Markus, L: Controllability of [r]-matrix quasi-differential equations. J. Differ. Equ. 89, 95-109 (1991)

doi:10.1186/1687-1847-2012-115

Cite this article as: Fang and Sun: Existence and uniqueness of solutions to complex-valued nonlinear impulsive

differential systems. Advances in Difference Equations 2012 2012:115.

\section{Submit your manuscript to a SpringerOpen ${ }^{\circ}$ journal and benefit from:}

- Convenient online submission

- Rigorous peer review

- Immediate publication on acceptance

- Open access: articles freely available online

- High visibility within the field

- Retaining the copyright to your article

Submit your next manuscript at $>$ springeropen.com 